\title{
The prognostic role of plasma fibrinogen in adult secondary hemophagocytic lymphohistiocytosis
}

\author{
Guangli Yin ${ }^{1}$, Changfeng Man¹, Jiayu Huang ${ }^{1}$, Shengen Liao², Xin Gao ${ }^{3}$ Tian Tian³, Limin Duan³ , Ji Xu³ \\ and Hongxia Qiu ${ }^{1,3^{*}}$ (D)
}

\begin{abstract}
Background: In adult patients with secondary hemophagocytic lymphohistiocytosis (sHLH), no valid immune biomarker has been available for predicting the prognosis of untreated sHLH patients.

Methods: Circulating plasma levels of fibrinogen (FIB) were measured at diagnosis in 293 cases of adult sHLH. We categorized FIB levels into tertiles. Multivariable Cox proportional hazards models were used to evaluate the relationship between FIB and survival. Restricted cubic spline models and two-piecewise Cox proportional hazards models were used to address the nonlinear association between FIB and mortality.

Results: During a median follow-up of 52 (interquartile ranges, 18-221) days, 208 deaths occurred, with 137 deaths in malignancy-associated hemophagocytic lymphohistiocytosis (MHLH) and 71 deaths in non-malignancy-associated hemophagocytic lymphohistiocytosis (non-MHLH). After multivariable adjustment, compared with the highest tertile of FIB, the hazard ratios (HRs) with 95\% confidence intervals (Cls) of survival for tertile 2 and tertile 1 were 1.06 $(0.90-1.24)$ and $0.84(0.71-0.98)$, respectively. The restricted cubic spline curve displayed a nonlinear and inverse relationship between FIB and mortality. Furthermore, the threshold effect analysis demonstrated that the inflection point for the curve was at an FIB level of $1.76 \mathrm{~g} / \mathrm{L}$. The HRs (95\% Cls) for survival were $0.68(0.55-0.83)$ and $1.08(0.96-1.21)$ on the left and right side of the inflection point, respectively.
\end{abstract}

Conclusions: These results suggest that plasma fibrinogen is nonlinearly and inversely associated with the risk of mortality in adult secondary hemophagocytic lymphohistiocytosis.

Keywords: Hemophagocytic lymphohistiocytosis, Fibrinogen, Prognostic, Nonlinear, Mortality

\section{Background}

Adult secondary hemophagocytic lymphohistiocytosis (sHLH) is a condition of pathologic immune dysregulation characterized by uncontrolled activation of cytotoxic $\mathrm{T}$ lymphocytes and macrophages, producing abnormal hypercytokinemia and leading to multiple

\footnotetext{
*Correspondence: qhx9805@126.com

1 Department of Hematology, The First Affiliated Hospital of Nanjing Medical University, Jiangsu Province Hospital, 300 Guangzhou Road, Nanjing 210029, China

Full list of author information is available at the end of the article
}

organ failure [1]. These abnormal cytokines are responsible for variable clinical symptoms and signs, such as fever, profound pancytopenia, organomegaly (including lymphadenopathy, hepatomegaly, and splenomegaly), liver dysfunction, hypertriglyceridemia, hyperferritinemia, central nervous system (CNS) symptoms, dermatologic abnormalities, and coagulation disorders, that may result in high morbidity and mortality [2]. In the previous studies focusing on outcomes, mortality ranged from 20.4 to $88 \%$ depending on the underlying disease and triggering condition, including 
malignancies, infections, and autoimmune disorders [3]. Early identification of the severity of adult HLH and prognosis would be helpful for enhancing the patients' chances of survival; therefore, improved prognostic indicators are needed.

Coagulation disorders are common in sHLH patients and are reported in up to $60 \%$ of patients with sHLH [4]. Fibrinogen (FIB) less than or equal to $1.50 \mathrm{~g} / \mathrm{L}$, one of the most frequently reported coagulopathies and the hallmark of HLH, is also a diagnostic criterion for HLH [5]. Several studies have also demonstrated that $50-80 \%$ of sHLH patients have hypofibrinogenemia $[6,7]$. Recently, one pediatric HLH study reported that plasma FIB levels $(<150 \mathrm{mg} / \mathrm{dL})$ at diagnosis may be prognostic measures of inferior survival [8]. Several retrospective studies have reported the prognostic role of plasma fibrinogen in adult sHLH $[4,9]$. However, these studies were limited by a relatively small sample size $(<120$ patients) and lacked follow-up data or adjusted confounding factors influencing prognosis for sHLH patients $[9,10]$. In addition, no quantitative analyses were performed to address the dose-response relationship between FIB and the risk of mortality, and whether a linear or nonlinear relationship exists between FIB and the risk of mortality is still unknown.

Therefore, whether reduced FIB levels are associated with poor survival in adult $\mathrm{sHLH}$ independent of conventional risk factors currently remains unclear. Thus, we aimed to explore whether reduced FIB levels independently affected long-term survival in a retrospective cohort study with SHLH.

\section{Methods \\ Study patients}

A total of 293 consecutive patients with a primary diagnosis of adult secondary hemophagocytic lymphohistiocytosis (sHLH) between January 1, 2014, and January 1, 2020, were enrolled. The HLH-2004 criteria developed by Henter et al. [11] and the HScore by Fardet et al. [12] were routinely used to help with the diagnosis of HLH. The inclusion of patients was based on (1) age $\geq 18$ years and (2) fulfillment of at least 5 of the 8 criteria proposed by the Histiocyte Society in 2004. The exclusion criteria included (1) Patients less than 18 years old $(n=5)$; (2) patients with a history of anticoagulant therapy $(n=6)$; and (3) patients with a history of cirrhosis or other serious liver diseases $(n=4)$. Figure 1 shows the flow chart of the patient inclusion and exclusion process in this study. Our study was approved by the ethics committee of the First Affiliated Hospital of Nanjing Medical University (Clinical Trial: ChiCTR2000032421). The study was conducted in accordance with the Declaration of Helsinki. Informed consent was obtained to review patient medical records.

\section{Clinical data}

Patients' histories and clinical characteristics including age; gender; fever; complete blood cell counts

A total of 326 consecutive sHLH patients in our study were admitted to First Affiliated Hospital of Nanjing Medical University between January 1, 2014, and January 1, 2020

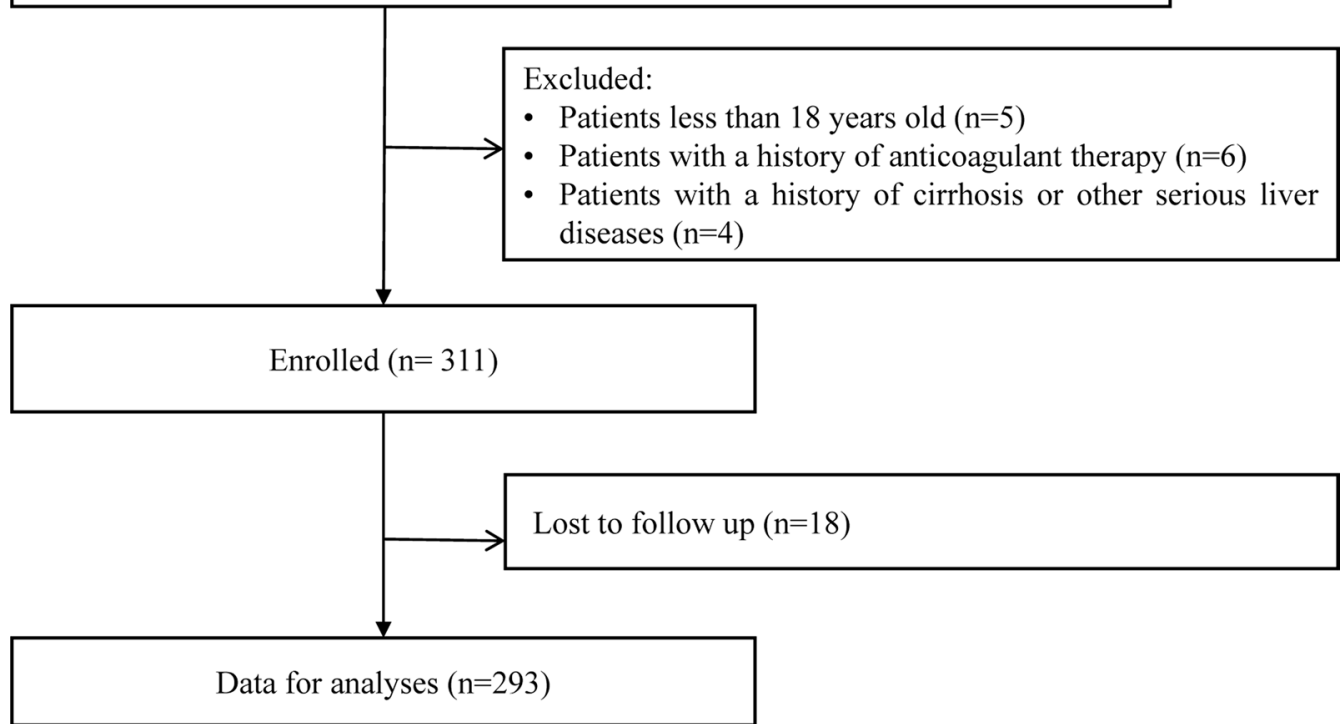

Fig. 1 Study flow chart. SHLH secondary hemophagocytic lymphohistiocytosis 
(CBC); hepatosplenomegaly; and blood biochemical tests (including aspartate aminotransferase (AST), alanine aminotransferase (ALT), triglycerides (TG), lactate dehydrogenase (LDH), fibrinogen (FIB), ferritin, serum soluble interleukin-2 receptor (sIL-2R, sCD25), and $\beta_{2}$-microglobulin ( $\beta_{2}$-MG) tests) were reviewed from their medical records on admission. Epstein-Barr virus (EBV) was evaluated by both serology and EBV DNA real-time quantitative polymerase chain reaction (RQ-PCR) analysis. The NK-cell cytotoxicity assay is not available at our facility. Bone marrow aspiration and biopsy samples were reviewed at the first diagnosis. Tumor or lymph node biopsy and PET/CT confirmed malignancy-associated hemophagocytic lymphohistiocytosis (MHLH).

The FIB levels were measured in the clinical laboratory of our hospital following regular procedures and assayed by the Clauss method with an automatic coagulometer (Sysmex CS5100, Japan). The FIB levels were categorized in tertiles according to the distributions of the study population: tertile $1(\mathrm{~T} 1) \leq 1.20,1.21 \leq$ tertile $2(\mathrm{~T} 2) \leq 1.97$ and tertile $3(\mathrm{~T} 3)>1.97$.

\section{Follow-up and endpoints}

The primary outcome of the current study was overall survival (OS), which was calculated as the time in days from sHLH diagnosis to the date of death from any cause or the last follow-up. The survival status of all participants was confirmed with death records or a telephone call to the patient's relatives or to the patient themselves and ascertained by checking government records of death.

\section{Treatment according to causes of $\mathrm{SHLH}$}

Two hundred and seventy-eight patients (95.2\%) received a specific treatment for sHLH. In our 169 MHLH patients, 120 patients had received systemic combination chemotherapy as the first-line therapy for sHLH; 33 patients were treated with HLH-94 as the initial therapy; 10 patients with progressive multiple organ dysfunction were treated with only intravenous immunoglobulins (IVIg) and glucocorticoid (GC). In our 124 non-malignancy-associated hemophagocytic lymphohistiocytosis (non-MHLH) patients, first-line treatment using HLH-94 was administered in 14 patients; GC was administered in 11 patients; GC + IVIg was administered in 46 patients; GC+etoposide was administered in 26 patients; GC+IVIg + cyclosporine was administered in 15 patients; and GS + IVIg + cyclophosphamide was administered in 4 patients. No difference in treatment regimens occurred across the FIB groups.

\section{Statistical analysis}

Continuous variables were presented as the means with standard deviations (SDs) for normally distributed variables or the median with the interquartile range (IQR) for nonnormally distributed variables, and categorical variables were described as numbers and proportions (percentages). Differences across FIB tertiles were analyzed using one-way ANOVA and the Kruskal-Wallis and chi-squared tests where appropriate. We used univariate and multivariable Cox proportional hazards models to estimate hazard ratios (HRs) and 95\% CIs to examine the relationship between fibrinogen concentrations and survival (variables with $P<0.05$ in the univariate Cox regression were subsequently entered into the multivariable model). The fibrinogen of T3 was used as a reference to estimate HR and 95\% CI. KaplanMeier curves for tertiles of fibrinogen were plotted to show mortality rates. Survival curves were adjusted for covariates derived from the final model according to multivariable Cox proportional hazards models.

A restricted cubic spline (with 3 knots located at the 10th, 50th, and 90th percentiles; the number of knots was selected according to the Akaike information criterion) was conducted to address the nonlinear relationship between fibrinogen and the risk of mortality after adjusting for confounding factors. If nonlinearity was detected, we calculated the inflection point using a recursive algorithm and then constructed a two-piecewise Cox proportional hazards model on both sides of the inflection point. The threshold level was calculated according to the inflection point with the maximum model likelihood using the trial and error method. A log likelihood ratio test comparing the one-line Cox proportional hazards model with a two-piecewise Cox proportional hazards model was performed to examine the statistical significance.

Stratified analyses were conducted according to gender (male or female), age ( $\leq 60$ years or $>60$ years), ferritin $\quad(\leq 10,000 \quad \mu \mathrm{g} / \mathrm{L} \quad$ or $>10,000 \quad \mu \mathrm{g} / \mathrm{L}), \quad s C D 25$ $(\leq 20,000 \mathrm{ng} / \mathrm{L}$ or $>20,000 \mathrm{ng} / \mathrm{L}$ ), EBV status (no EBV infection or EBV infection), and etiologies (MHLH or non-MHLH). We tested for potential effect modification by these stratification variables by including interaction terms between fibrinogen and a potential effect modifier (overall survival) in the multivariate adjusted model and by conducting a likelihood ratio test (LRT) comparing the models with and without interaction terms. Tests for trends were calculated by including the median value for each corresponding tertile as a continuous variable in the models [13]. All statistical analyses were conducted with $\mathrm{R}$ software (version 3.6.0; The $\mathrm{R}$ Foundation for Statistical Computing) and STATA statistical software (version 14.0; StataCorp, TX, USA). 
A two-sided $P<0.05$ was considered statistically significant for all analyses.

\section{Results}

\section{Distribution of plasma fibrinogen and patient} characteristics

The baseline characteristics for patients stratified by FIB tertiles are listed in Table 1.The mean age of the total study population was 53 (41-64) years, and $62.5 \%$ were male. The median FIB level in the overall population was $1.50 \mathrm{~g} / \mathrm{L}$ (IQR $1.02-2.18 \mathrm{~g} / \mathrm{L}$ ). The participants with lower FIB had lower platelet levels and higher ALT, AST, LDH, TG, $\beta_{2}$-MG, serum ferritin, and sCD25 levels and total Hscore points and were more likely to be infected with EBV (all $P<0.05$ ). Detailed etiological classification of sHLH patients was shown in Additional file 1.

\section{Associations of the plasma fibrinogen with survival}

The median follow-up duration was 52 (IQR, 18-221) days. The follow-up found 208 deaths, including 137 deaths in MHLH and 71 deaths in non-MHLH. Figure 2 shows the survival curves with regard to different categories of FIB in the total patients for cumulative overall survival. Patients with T3 and T2 had a similar survival $(P=0.726)$, whereas patients with $\mathrm{T} 1$ had a significantly worse survival $(\mathrm{HR}=0.76,95 \% \mathrm{CI} 0.66-0.87 ; P<0.001)$ than those with T3. After adjusting for confounding factors, including age, PLT, ferritin, EBV infection, MHLH, and treatment remedies, the HRs with $95 \%$ CIs of survival for T2 and T1 were $1.06(0.90-1.24)$ and $0.84(0.71-$ 0.98 ), respectively ( $P$ for trend: 0.04 ) (Table 2 and Fig. 2 ). This finding was also in line with MHLH and non-MHLH group (Fig. 3).

Table 1 Baseline demographic, clinical, and laboratory characteristics of the study patients according to tertiles of fibrinogen

\begin{tabular}{|c|c|c|c|c|c|}
\hline & Total $(\mathrm{N}=293)$ & Tertile $1(\mathrm{~N}=99)$ & Tertile $2(\mathrm{~N}=97)$ & Tertile $3(\mathrm{~N}=97)$ & $P$ \\
\hline $\mathrm{FIB}, \mathrm{g} / \mathrm{L}$ & $1.50(1.02-2.18)$ & $0.86(0.70-1.03)$ & $1.51(1.38-1.72)$ & $2.59(2.18-3.33)$ & $<0.001$ \\
\hline Male, n (\%) & $183(62.5)$ & $62(62.6)$ & $60(61.9)$ & $61(62.9)$ & 0.988 \\
\hline Age, years & $53(41-64)$ & $51(33-64)$ & $56(39-62)$ & $55(46-66)$ & 0.118 \\
\hline $\mathrm{ANC}, \times 10^{9} / \mathrm{L}$ & $1.32(0.69-2.35)$ & $1.04(0.57-2.11)$ & $1.36(0.77-2.24)$ & $1.55(0.81-2.71)$ & 0.057 \\
\hline $\mathrm{HB}, \mathrm{g} / \mathrm{L}$ & $86(71-100)$ & $84(77-95)$ & $90(73-107)$ & $82(68-97)$ & 0.111 \\
\hline $\mathrm{PLT}, \times 10^{9} / \mathrm{L}$ & $43(24-72)$ & $31(18-52)$ & $45(25-80)$ & $54(32-97)$ & $<0.001$ \\
\hline$A L T, U / L$ & $67.1(36.3-149.1)$ & $98.6(50.6-235.4)$ & 77.6 (32.4-143.2) & $50.1(28.4-82.3)$ & $<0.001$ \\
\hline AST, U/L & $91(46.1-203.8)$ & $178.5(78.1-411.0)$ & $90.2(45.1-198.1)$ & $61.0(39.5-121.65)$ & $<0.001$ \\
\hline $\mathrm{LDH}, \mathrm{U} / \mathrm{L}$ & $676(394.0-1308.5)$ & $1004(580.0-1793.0)$ & $646(354.5-1101.0)$ & $540(379.5-934.0)$ & $<0.001$ \\
\hline $\mathrm{TG}, \mathrm{mmol} / \mathrm{L}$ & $2.47(1.71-3.70)$ & $2.78(1.77-4.04)$ & $2.52(1.89-3.67)$ & $2.01(1.58-3.18)$ & 0.015 \\
\hline Ferritin, ug/L & $4739(1522-12,798)$ & $8133(2000-20,000)$ & $3639(1613-9558)$ & $2841(1494-7095)$ & $<0.001$ \\
\hline sCD25, ng/L & $33,480(17,442-51,668)$ & $40,042(22,660-55,919)$ & $26,920(14,266-48,289)$ & $32,939(16,018-47,762)$ & 0.024 \\
\hline$\beta_{2}-M G, m g / L$ & $5.97(4.41-8.53)$ & $5.91(4.19-8.53)$ & $7.63(4.85-9.28)$ & $5.69(3.88-8.04)$ & 0.030 \\
\hline Fever, ${ }^{\circ} \mathrm{C}$ & $39.5(39.0-40.0)$ & $39.6(39.0-40.0)$ & $39.4(39.0-40.0)$ & $39.3(39.0-40.0)$ & 0.143 \\
\hline Splenomegaly (\%) & $260(88.7)$ & $90(90.9)$ & $88(90.7)$ & $82(84.5)$ & 0.278 \\
\hline Lymphadenopathy (\%) & $152(51.9)$ & $45(45.5)$ & $52(53.6)$ & $55(56.7)$ & 0.265 \\
\hline Hemophagocytic (\%) & $260(88.7)$ & $88(88.9)$ & 85 (87.6) & $87(89.7)$ & 0.900 \\
\hline HScore, points & $234(205-269)$ & $255(224-283)$ & 235 (210-274) & $219(179-244)$ & $<0.001$ \\
\hline EBV infection (\%) & $148(50.5)$ & $65(65.7)$ & $44(45.4)$ & $39(40.2)$ & 0.001 \\
\hline Etiology & & & & & 0.120 \\
\hline MHLH (\%) & $169(57.7)$ & $65(65.7)$ & $50(51.5)$ & $54(55.7)$ & \\
\hline Non-MHLH (\%) & $124(42.3)$ & $34(34.3)$ & $47(48.5)$ & $43(44.3)$ & \\
\hline Treatment & & & & & 0.960 \\
\hline Chem \pm HLH-94 (\%) & $167(57.0)$ & $58(58.6)$ & $53(54.6)$ & $56(57.7)$ & \\
\hline $\mathrm{GC} \pm \mathrm{IVIG} \pm \mathrm{CsA} \pm \mathrm{VP} 16(\%)$ & $112(38.2)$ & $36(36.4)$ & $40(41.2)$ & $36(37.1)$ & \\
\hline Support (\%) & $14(4.8)$ & $5(5.1)$ & $4(4.1)$ & $5(5.2)$ & \\
\hline
\end{tabular}

Italics indicate statistical significance $(P<0.05)$

FIB fibrinogen, $A N C$ absolute neutrophil count, $H B$ hemoglobin, $P L T$ platelet, $A L T$ alanine transaminase, $A S T$ aspartate transaminase, $L D H$ lactate dehydrogenase, $A L B$ albumin, TG triglyceride, $s C D 25$ soluble interleukin-2 receptor, $\beta_{2}-M G$ beta ${ }_{2}$-microglobulin, EBV Epstein-Barr virus, $M H L H$ malignancy-associated haemophagocytic lymphohistiocytosis, Non-MHLH non-malignancy associated haemophagocytic lymphohistiocytosis, GC glucocorticoid, IVIg intravenous immunoglobulins, CsA cyclosporine, VP16 etoposide 

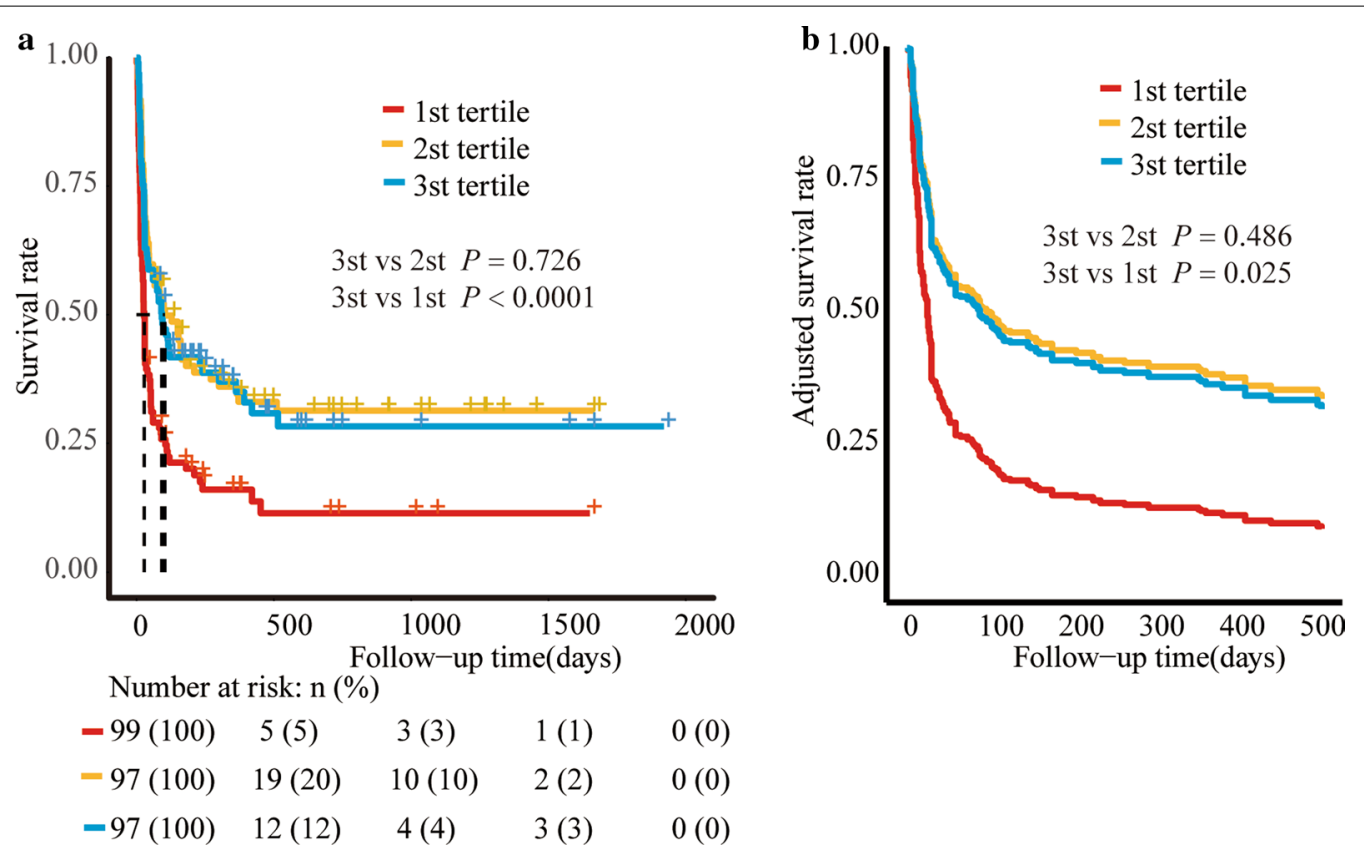

The associations between FIB (continuously) and the risk of mortality are shown in Fig. 4. The fully adjusted cubic curve fitting showed that FIB was nonlinearly and inversely associated with the risk of death, and the tests for nonlinearity were significant ( $P$ for nonlinearity $<0.001$ ). We further conducted a threshold effect analysis of FIB on the survival. When we fitted the association between FIB and survival using a Cox proportional hazards model and a two-piecewise Cox proportional hazards model, the $P$ value for the log likelihood ratio test was less than 0.001 , indicating that the two-piecewise Cox proportional hazards model was more suitable for fitting the association between FIB and survival. The inflection point that we identified for FIB was $1.76 \mathrm{~g} / \mathrm{L}$. When FIB was $\leq 1.76 \mathrm{~g} / \mathrm{L}$, decreased FIB was significantly associated with an increased risk of poor survival (HR $=0.68,95 \%$ CI, 0.55-0.83; $P<0.001)$. Conversely, when FIB was $>1.76 \mathrm{~g} / \mathrm{L}$, the survival did not change significantly with increased FIB (HR $=1.08,95 \%$ CI, 0.96$1.21 ; P=0.187$ ) (Table 3$)$.

\section{Subgroup analysis}

The subgroup analyses on survival according to multiple confounding variables are shown in Table 4. Although no significant interactions for the associations between FIB and survival were observed across strata by age, sex, ferritin, sCD25, EBV infection and etiologies, participants who were younger, who were male, who had ferritin $\leq 10,000 \mu \mathrm{g} / \mathrm{L}$, who had a high level of $\mathrm{sCD} 25$, who had EBV infection, and who were non-MHLH were more likely to have a worse survival with a decrease in FIB.

\section{Discussion}

To our knowledge, this is the largest cohort study assessing the prognostic role of fibrinogen in adult sHLH. In addition, our study first indicated that plasma fibrinogen level is nonlinearly and inversely associated with poor survival in adult sHLH patients regardless of conventional confounding factors, including age, PLT, EBV infection, ferritin, etiologies and treatment strategies. Further threshold effect analysis showed that the inflection point was $1.76 \mathrm{~g} / \mathrm{L}$, and survival was inconsistent on the left and right sides of the inflection point.

A few previous studies regarding the association of FIB and its threshold with the risk of mortality defined plasma FIB levels using different categories or cut-off values. In pediatric patients with HLH $[8,14]$, Signoff demonstrated that hypofibrinogenemia $(\mathrm{FIB}<1.5 \mathrm{~g} / \mathrm{L}$ ) was independently associated with higher mortality rates (adjusted odds ratio, 6.0; 95\% CI, 2.0-18.1); meanwhile, in adult sHLH studies, Sandrine et al. [4] used 117 ICU patients and found that coagulation disorders were associated with higher mortality, especially $\mathrm{FIB}<2 \mathrm{~g} / \mathrm{L}$ (adjusted $\mathrm{OR}=2.42,95 \%$ CI $1.08-5.41 ; P=0.04$ ). The large sample size of this study combined with the 
Table 2 Uni- and multi-variate Cox regression analyses of survival

\begin{tabular}{|c|c|c|c|c|}
\hline \multirow[t]{2}{*}{ Characteristics } & \multicolumn{2}{|l|}{ Unadjusted } & \multicolumn{2}{|l|}{ Adjusted } \\
\hline & HR $(95 \% \mathrm{Cl})$ & $P$ & HR $(95 \% \mathrm{Cl})$ & $P$ \\
\hline Male & $0.85(0.75-0.96)$ & 0.010 & & \\
\hline Age, years & $1.00(0.99-1.00)$ & 0.009 & $1.00(0.99-1.00)$ & 0.017 \\
\hline ANC $>1.0 \times 109 / \mathrm{L}$ & $0.94(0.84-1.06)$ & 0.331 & & \\
\hline $\mathrm{HB}<90 \mathrm{~g} / \mathrm{L}$ & $0.87(0.77-0.98)$ & 0.027 & & \\
\hline $\mathrm{PLT}<100 \times 10^{9} / \mathrm{L}$ & $0.76(0.62-0.91)$ & 0.004 & $0.81(0.66-0.99)$ & 0.043 \\
\hline $\mathrm{LDH} \geq 2.5 \times \mathrm{ULN}$ & $1.14(0.93-1.39)$ & 0.205 & & \\
\hline $\mathrm{TG} \geq 3.0 \mathrm{mmol} / \mathrm{L}$ & $0.93(0.82-1.05)$ & 0.245 & & \\
\hline Fever, $\geq 39.1{ }^{\circ} \mathrm{C}$ & $1.02(0.90-1.15)$ & 0.775 & & \\
\hline Splenomegaly & $0.95(0.78-1.16)$ & 0.636 & & \\
\hline Hemophagocytosis & $1.08(0.9-1.29)$ & 0.403 & & \\
\hline $\log _{10}$ (Ferritin) & $0.75(0.66-0.85)$ & $<0.001$ & $0.78(0.68-0.90)$ & 0.001 \\
\hline $\log _{10}(\mathrm{sCD} 25)$ & $0.77(0.65-0.92)$ & 0.003 & & \\
\hline EBV infection & $0.78(0.69-0.88)$ & $<0.001$ & $0.85(0.74-0.96)$ & 0.011 \\
\hline MHLH & $0.78(0.69-0.88)$ & $<0.001$ & $0.63(0.54-0.74)$ & $<0.001$ \\
\hline \multicolumn{5}{|l|}{ Treatment } \\
\hline $\begin{array}{l}\text { Support treat- } \\
\text { ment }\end{array}$ & 1.00 (Ref.) & & 1.00 (Ref.) & \\
\hline $\mathrm{GC}+\mathrm{IVIG}$ & $0.90(0.73-1.11)$ & 0.323 & $1.08(0.87-1.35)$ & 0.462 \\
\hline Chem \pm HLH94 & $1.15(0.93-1.40)$ & 0.196 & $1.74(1.38-2.22)$ & $<0.001$ \\
\hline \multicolumn{5}{|l|}{$\mathrm{FIB}(\mathrm{g} / \mathrm{L})$} \\
\hline 3 st tertile & 1.00 (Ref.) & & 1.00 (Ref.) & \\
\hline 2 st tertile & $1.03(0.88-1.20)$ & 0.726 & $1.06(0.90-1.24)$ & 0.486 \\
\hline 1 st tertile & $0.76(0.66-0.87)$ & $<0.001$ & $0.84(0.71-0.98)$ & 0.025 \\
\hline
\end{tabular}

Italics indicate statistical significance $(P<0.05)$

$A N C$ absolute neutrophil count, $H B$ hemoglobin, $P L T$ platelet, $L D H$ lactate dehydrogenase, $T G$ triglyceride, $S C D 25$ soluble interleukin-2 receptor, EBV Epstein-Barr virus, $M H L H$ malignancy-associated haemophagocytic lymphohistiocytosis, GC glucocorticoid, IVIg intravenous immunoglobulins, FIB fibrinogen, $H R$ hazard ratio, $\mathrm{Cl}$ confidence interval

application of RCS afforded us greater statistical power and model flexibility to characterize the associations of plasma FIB concentrations with mortality.

The increased risk of mortality associated with low fibrinogen might be ascribed to the activation of lymphocyte and macrophage immune cells, creating an uncontrolled loop of inflammation that is responsible for liver dysfunction, DIC and fibrinolysis. Stimulated macrophages secrete proinflammatory cytokines, including TNF alpha, IL-1beta, and IL-6, which in turn can release tissue plasminogen activator in excess; correspondingly, an increase in plasmin responsible for fibrinolysis leads to low fibrinogen levels $[15,16]$. In addition, high IFN- $\gamma$, presented in $\mathrm{sHLH}$, can induce the production of tissue factor expression in activated macrophages, and activated histiocytes can activate factor X through Mac-1 receptors, eventually initiating blood coagulation and aberrantly triggering an overconsumption of fibrinogen [17, 18]. Another mechanism could be diffuse liver infiltration with activated $\mathrm{T}$ lymphocytes and macrophages and hypercytokinemia [19]. All of the above mechanisms, alone or together, may be attributed to low fibrinogen levels, which reflect low in vivo disease activity. Hypofibrinogenemia was also indicative of adverse outcomes beyond coagulopathy, as indicated by its persistent association with a complicated course and sHLH/MAS with hyperinflammation.

Our study explored the nonlinear association between plasma FIB levels and the risk of mortality and indicated that FIB displayed an L-shaped relationship with the risk of mortality. One hypothesis is that the lower fibrinogen level $(\leq 1.76 \mathrm{~g} / \mathrm{L})$ is only the reflection of a more severe form of HLH (with more intense cytokine storm and intense hemophagocytic activity). These patients with lower fibrinogen levels who have worse survival may reach a certain threshold of excessive activated immune cells and overwhelming systemic inflammation. Early identification of these patients may prompt earlier consideration of alternative therapeutic strategies, including intensive immunotherapies.

Our study has several strengths. This is the first study to address the nonlinearity between FIB levels at the diagnosis of adult $\mathrm{sHLH}$ and the risk of mortality and further explains this nonlinearity with a threshold effect analysis. However, several limitations of our study were noted. First, due to the single-center retrospective study, the results may not be generalizable to a broader population. Second, although we had fully adjusted a broad set of covariates available to influence prognosis, we could not rule out the possibility that our findings were biased by unmeasured or unrecognized confounders. Finally, the observational study could only demonstrate the association between FIB levels and the prognosis of patients with sHLH; it could not provide conclusions regarding causality.

\section{Conclusion}

Our study demonstrated that plasma FIB levels are nonlinearly and inversely associated with poor survival in adult sHLH patients. The threshold effect of FIB on poor survival was $1.76 \mathrm{~g} / \mathrm{L}$. Each 1 unit decrease in FIB was associated with a $32 \%$ decrease in survival when the serum FIB levels were $\leq 1.76 \mathrm{~g} / \mathrm{L}$. 

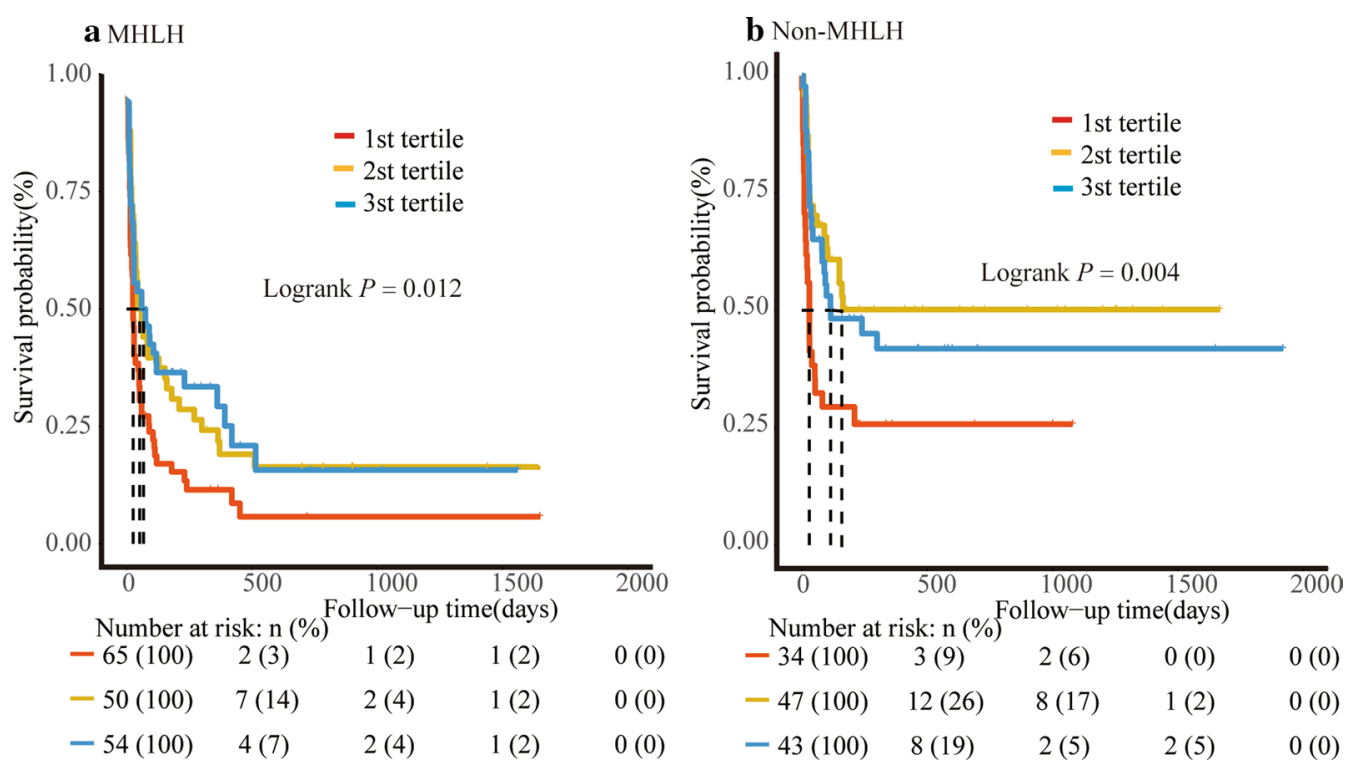

Fig. 3 Survival curves of sHLH patients according to fibrinogen tertiles. a MHLH; b non-MHLH. MHLH malignancy-associated HLH, non-MHLH non-malignancy-associated hemophagocytic lymphohistiocytosis

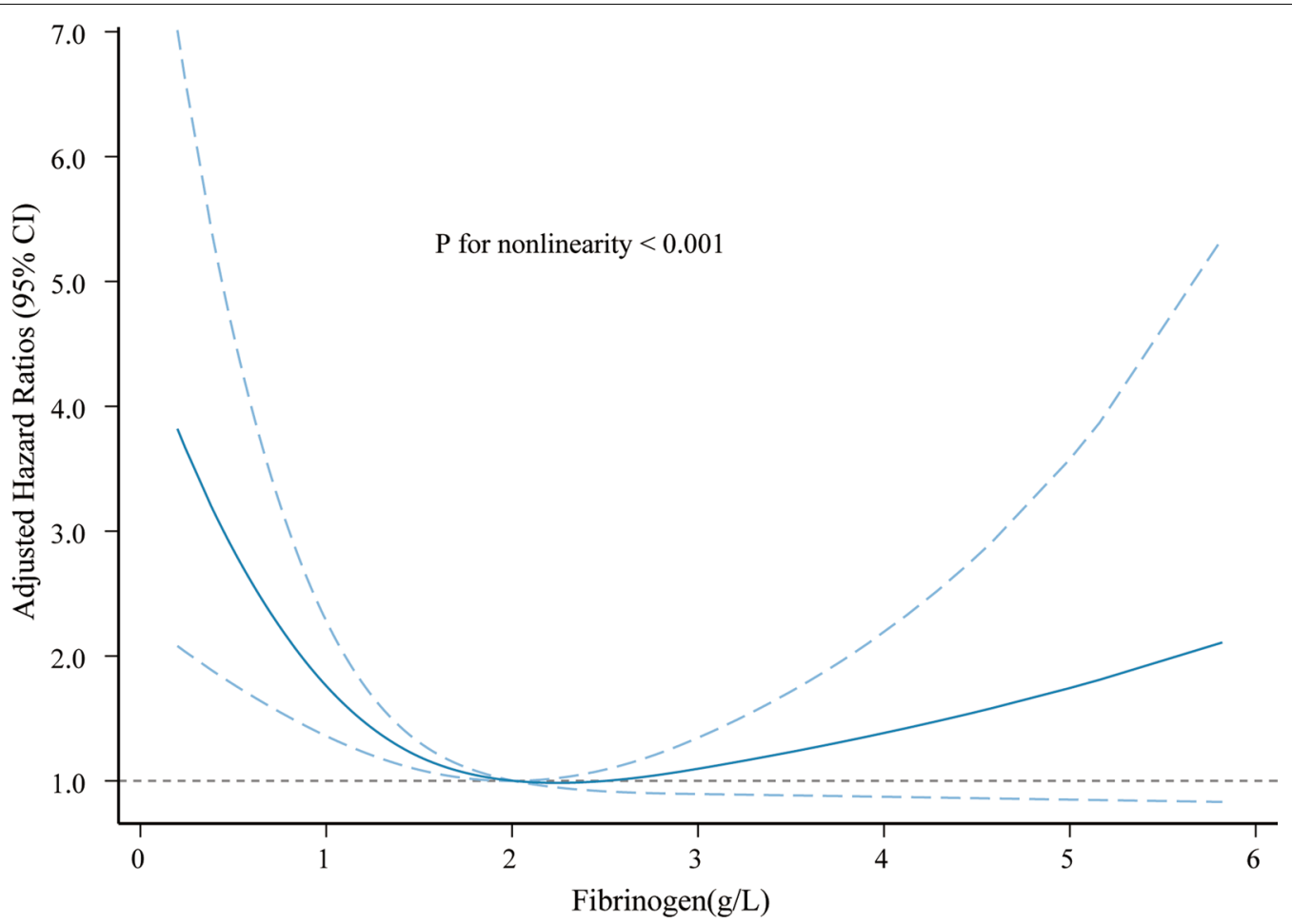

Fig. 4 Cubic spline plot of the association between plasma fibrinogen and the risk of mortality among adult onset HLH patients. The solid line and dashed line represent the estimated hazard ratios and their corresponding $95 \% \mathrm{Cls}$, respectively. Analyses were adjusted for age, PLT (platelet), EBV, ferritin, malignancy —associated HLH (MHLH) and treatment strategies 
Table 3 Threshold effect analysis of fibrinogen on survival using two-piecewise Cox proportional hazards regression models

\begin{tabular}{lllcc}
\hline Inflection point & Group & HR $(\mathbf{9 5} \% \mathrm{Cl})$ & $P$ value & $P$ for log likelihood ratio test \\
\hline 1.76 & $\leq 1.76$ & $0.68(0.55-0.83)$ & $<0.001$ & $<0.001$ \\
& $>1.76$ & $1.08(0.96-1.21)$ & 0.187 & \\
\hline
\end{tabular}

Analyses were adjusted for age, PLT (platelet), EBV, log10-transform ferritin, MHLH and treatment strategies

$H R$ hazard ratio, $\mathrm{Cl}$ confidence interval

Table 4 Stratified associations between fibrinogen and survival

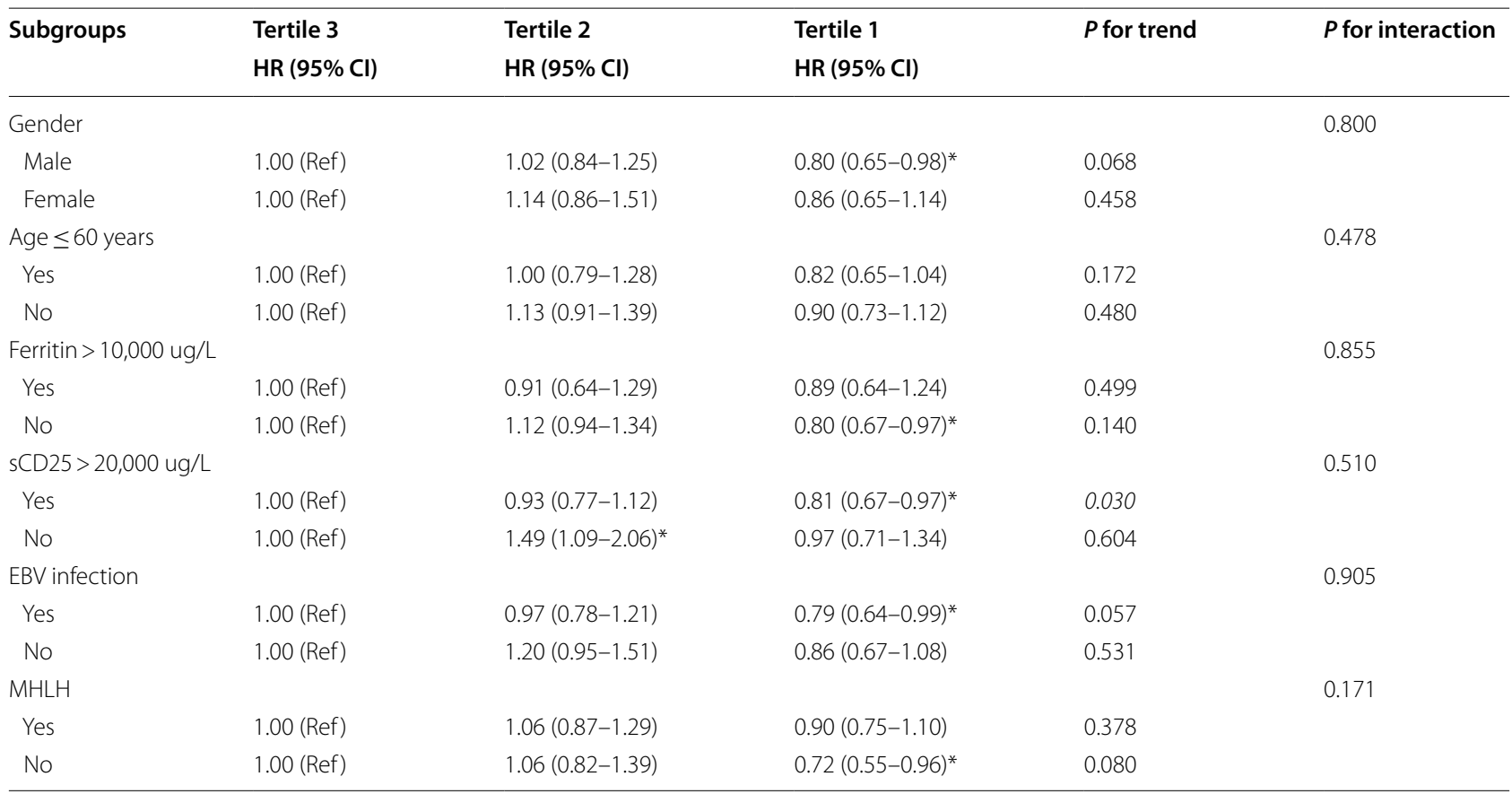

Analyses were adjusted for age, PLT (platelet), EBV, $\log _{10}$-transform ferritin, $\mathrm{MHLH}$ and treatment strategies when they were not stratified variables Italics indicate statistical significance $(P<0.05)$

$H R$ hazard ratio, $\mathrm{Cl}$ confidence interval

${ }^{*} P<0.05 ;{ }^{*} P<0.001$

\section{Supplementary information}

Supplementary information accompanies this paper at https://doi. org/10.1186/s13023-020-01622-2.

Additional file 1. Detailed etiological classification of 293 adult sHLH (MHLH and Non-MHLH).

\section{Abbreviations}

SHLH: Secondary hemophagocytic lymphohistiocytosis; FIB: Fibrinogen; ANC: Absolute neutrophil count; HB: Hemoglobin; PLT: Platelet; ALT: Alanine transaminase; AST: Aspartate transaminase; LDH: Lactate dehydrogenase; TG: Triglyceride; $5 C D 25$ : Soluble interleukin-2 receptor; $\beta_{2}-M G$ : Beta 2 -microglobulin; EBV: Epstein-Barr virus; MHLH: Malignancy-associated hemophagocytic lymphohistiocytosis; Non-MHLH: Non-malignancy associated hemophagocytic lymphohistiocytosis; GC: Glucocorticoid; IVlg: Intravenous immunoglobulins; CsA: Cyclosporine; VP16: Etoposide; HR: Hazards ratio; 95\% Cl: 95\% Confidence interval.

\section{Acknowledgements}

The authors express their gratitude for all persons who were involved in reporting on patients and gathering data.

\section{Authors' contributions}

GLY and HXQ designed the experiments. GLY performed the experiments. GLY, CFM and HXQ organized the clinical materials. GLY and SGL performed the data analysis. GLY wrote the paper. All authors contributed to the final approval of the manuscript. All authors read and approved the final manuscript.

\section{Funding}

This study was supported by the National Natural Science Foundation of China (Grant Number 81570175)

\section{Availability of data and materials}

The data of our patients is available in the Department of Medical Records at Jiangsu Province Hospital and the First Affiliated Hospital of Nanjing Medical University. These data can be released with consent from the patients and are available from the corresponding author upon reasonable request. 
Ethics approval and consent to participate

Our study was approved by the ethics committee of the First Affiliated Hospital of Nanjing Medical University and registered on the Chinese Clinical Trial Registry (ChiCTR2000032421).

\section{Consent for publication}

Consent for publication was obtained from all participants.

\section{Competing interests}

The authors declare that they have no competing interests.

\section{Author details}

1 Department of Hematology, The First Affiliated Hospital of Nanjing Medical University, Jiangsu Province Hospital, 300 Guangzhou Road, Nanjing 210029, China. ${ }^{2}$ Department of Cardiology, The First Affiliated Hospital of Nanjing Medical University, Jiangsu Province Hospital, 300 Guangzhou Road, Nanjing 210029, China. ${ }^{3}$ Department of Geriatric Hematology, The First Affiliated Hospital of Nanjing Medical University, Jiangsu Province Hospital, 300 Guangzhou Road, Nanjing 210029, China.

Received: 5 July 2020 Accepted: 16 November 2020

Published online: 25 November 2020

\section{References}

1. Al-Samkari H, Berliner N. Hemophagocytic lymphohistiocytosis. Annu Rev Pathol. 2018;13:27-49.

2. La Rosee P, Horne A, Hines M, von Bahr GT, Machowicz R, Berliner N, et al. Recommendations for the management of hemophagocytic lymphohistiocytosis in adults. Blood. 2019;133:2465-77.

3. Hayden A, Park S, Giustini D, Lee AY, Chen LY. Hemophagocytic syndromes (HPSs) including hemophagocytic lymphohistiocytosis $(\mathrm{HLH})$ in adults: a systematic scoping review. Blood Rev. 2016;30:411-20.

4. Valade S, Azoulay E, Galicier L, Boutboul D, Zafrani L, Stepanian A et al. Coagulation disorders and bleedings in critically ill patients with hemophagocytic lymphohistiocytosis. Med (Baltim). 2015;94:e1692.

5. Jordan MB, Allen CE, Greenberg J, Henry M, Hermiston ML, Kumar A, et al. Challenges in the diagnosis of hemophagocytic lymphohistiocytosis: recommendations from the North American Consortium for Histiocytosis (NACHO). Pediatr Blood Cancer. 2019;66:e27929.

6. Creput C, Galicier L, Buyse S, Azoulay E. Understanding organ dysfunction in hemophagocytic lymphohistiocytosis. Intensive Care Med 2008:34:1177-87.

7. Ramos-Casals M, Brito-Zeron P, Lopez-Guillermo A, Khamashta MA, Bosch X. Adult haemophagocytic syndrome. Lancet. 2014:383:1503-16.
8. Signoff JK, Fitzgerald JC, Teachey DT, Balamuth F, Weiss SL. Hypofibrinogenemia is associated with poor outcome and secondary hemophagocytic lymphohistiocytosis/macrophage activation syndrome in pediatric severe sepsis. Pediatr Crit Care Med. 2018;19:397-405.

9. Li F, Yang Y, Jin F, Dehoedt C, Rao J, Zhou Y, et al. Clinical characteristics and prognostic factors of adult hemophagocytic syndrome patients: a retrospective study of increasing awareness of a disease from a singlecenter in China. Orphanet J Rare Dis. 2015;10:20.

10. Park HS, Kim DY, Lee JH, Lee JH, Kim SD, Park YH, et al. Clinical features of adult patients with secondary hemophagocytic lymphohistiocytosis from causes other than lymphoma: an analysis of treatment outcome and prognostic factors. Ann Hematol. 2012;91:897-904.

11. Henter Jl, Horne A, Arico M, Egeler RM, Filipovich AH, Imashuku S, et al. HLH-2004: diagnostic and therapeutic guidelines for hemophagocytic lymphohistiocytosis. Pediatr Blood Cancer. 2007;48:124-31.

12. Fardet L, Galicier L, Lambotte O, Marzac C, Aumont C, Chahwan D, et al. Development and validation of the HScore, a score for the diagnosis of reactive hemophagocytic syndrome. Arthritis Rheumatol. 2014;66:2613-20

13. Liao S, Yao W, Cheang I, Tang X, Yin T, Lu X, et al. Association between perfluoroalkyl acids and the prevalence of hypertension among US adults. Ecotoxicol Environ Saf. 2020;196:110589.

14. Lee JY, Kim JH, Lee JS, Ryu JM, Seo JJ, Im HJ. Initial characteristics and clinical severity of hemophagocytic lymphohistiocytosis in pediatric patients admitted in the emergency department. Pediatr Emerg Care. 2018. https://doi.org/10.1097/PEC.0000000000001518.

15. Friesecke S, Stecher SS, Greinacher A. Tranexamic acid for treatment of bleeding in hemophagocytic lymphohistiocytosis. Thromb Res. 2015;135:1037-9.

16. Unkeless JC, Gordon S, Reich E. Secretion of plasminogen activator by stimulated macrophages. J Exp Med. 1974;139:834-50.

17. Kato J, Okamoto T, Motoyama H, Uchiyama R, Kirchhofer D, Van Rooijen N, et al. Interferon-gamma-mediated tissue factor expression contributes to T-cell-mediated hepatitis through induction of hypercoagulation in mice. Hepatology. 2013;57:362-72.

18. Ooe K. Pathogenesis of hypofibrinogenemia in familial hemophagocytic lymphohistiocytosis. Pediatr Pathol. 1991;11:657-61.

19. de Kerguenec C, Hillaire S, Molinie V, Gardin C, Degott C, Erlinger S, et al. Hepatic manifestations of hemophagocytic syndrome: a study of 30 cases. Am J Gastroenterol. 2001;96:852-7.

\section{Publisher's Note}

Springer Nature remains neutral with regard to jurisdictional claims in published maps and institutional affiliations.

Ready to submit your research? Choose BMC and benefit from:

- fast, convenient online submission

- thorough peer review by experienced researchers in your field

- rapid publication on acceptance

- support for research data, including large and complex data types

- gold Open Access which fosters wider collaboration and increased citations

- maximum visibility for your research: over $100 \mathrm{M}$ website views per year

At $\mathrm{BMC}$, research is always in progress.

Learn more biomedcentral.com/submissions 